\title{
Acute Anterior Cruciate Ligament Injury with Medial and Lateral Bucket-Handle Meniscus Tears
}

\author{
Joseph J. Fazalare, MD ${ }^{1,2,3}$ Jason Payne, MD $\quad$ Stephanie L. Stradley, PA ${ }^{3}$ Thomas M. Best, MD, PhD ${ }^{1,5}$ \\ Joseph Yu, MD ${ }^{4}$ David C. Flanigan, $\mathrm{MD}^{1,2,3}$
}

1 OSU Sports Medicine, The Ohio State University, Columbus, Ohio

2 The Sports Health and Performance Institute, The Ohio State University, Columbus, Ohio

${ }^{3}$ Department of Orthopaedics, The Ohio State University Medical Center, Columbus, Ohio

${ }^{4}$ Department of Radiology, The Ohio State University Medical Center, Columbus, Ohio

${ }^{5}$ Department of Family Medicine, The Ohio State University Medical Center, Columbus, Ohio

J Knee Surg Rep 2015;1:21-24.
Address for correspondence David C. Flanigan, MD, OSU Sports Medicine Center, 2050 Kenny Road, Suite 3100, Columbus, OH 432213502 (e-mail: david.flanigan@osumc.edu).

\begin{abstract}
Keywords

- meniscus

- ACL rupture

- bucket-handle meniscal tear

- MRI

We report the case of a healthy 31-year-old female professional billiard player presented with a 5-day history of severe left knee pain after a fall. A magnetic resonance imaging of the left knee showed that she had suffered an anterior cruciate ligament (ACL) rupture along with buckle-handle tears of both the medial and lateral meniscus. Both of these menisci had flipped anterior and centrally to the femoral condyles and were lodged in the notch. The patient had also suffered a mild injury to the medial collateral ligament. Repair of both menisci was performed using an inside-out technique. Following this, an $\mathrm{ACL}$ reconstruction was done using a quadrupled hamstring autograft. Endobutton fixation (Smith \& Nephew, Andover, MA) was used on the femur with a screw and sheath used for tibial fixation. The patient tolerated the procedure well and was sent home the same day. This case demonstrates the typical signs and symptoms of a displaced buckethandle meniscus tear as well as imaging and arthroscopic pictures.
\end{abstract}

A meniscus tear is a very common secondary injury seen in patients who have anterior cruciate ligament (ACL) ruptures. The lateral meniscus is more commonly injured in the setting of an acute ACL tear. ${ }^{1-3}$ One type of injury that is infrequently seen with ACL injuries is the bucket-handle meniscal tear (BHMT). A BHMT is a vertical or oblique longitudinal tear with an attached fragment displaced away from the periphery of the meniscus. ${ }^{4,5}$ BHMTs are quite rare and account for only $10 \%$ of all the meniscal lesions. ${ }^{4,5}$

The mechanism of BHMTs in the setting of ACL injuries can vary. ${ }^{6}$ For lateral BHMTs, the posterior aspect of the lateral meniscus typically gets trapped between the rotational impaction of the midportion of the lateral femoral condyle and posterior lateral tibial plateau (typical bone bruise pattern). As the knee reduces, the loose attachments of the posterior lateral meniscus around the popliteus hiatus tears further causing the meniscus to displace anteriorly and medially. Similarly, the medial meniscus can tear in both the acute and chronic setting causing a BHMT. In acute tears, a strong rotational force can tear the posterior capsular attachments causing the meniscus to displace anteriorly and laterally. The medial meniscus, which serves as a secondary stabilizer, can likewise be injured during episodes of instability and be torn in such a manner as to result in a medial-sided bucket-handle meniscus lesion. received

May 9, 2013

accepted after revision

July 12, 2014

published online

January 29, 2015
DOI http://dx.doi.org/

10.1055/s-0034-1399758. ISSN 2326-2729.
Copyright $\odot 2015$ by Thieme Medical Publishers, Inc., 333 Seventh Avenue, New York, NY 10001, USA. Tel: +1(212) 584-4662.
License terms

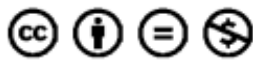


These tears are inherently unstable and can sometimes displace beyond the equator of the femoral condyle anteriorly and become lodged in the intercondylar notch. ${ }^{7}$ We present a patient with an acute ACL injury with both medial and lateral buckle-handle meniscus tears that were lodged in the intercondylar notch. To our knowledge, this bimeniscal injury has been reported only three other times in a younger patient $(<30$ years old $)$ who suffered an athletic injury. ${ }^{8-10}$ It has also been reported in an older athlete ${ }^{11}$ who suffered motor-vehicle trauma. ${ }^{12}$ The authors have obtained the patient's informed written consent for print and electronic publication of the case report.

\section{Case Report}

A healthy 31-year-old female professional billiard player presented to our office with a 5-day history of severe left knee pain. She had been standing on a small, 3-foot highplatform performing home maintenance projects. Subsequently, the patient lost her balance, fell, and suffered a violent twisting type injury to her knee. She felt and heard a "pop" in her knee during the injury. She had an immediate effusion with complaints of a mechanical block to range of motion. She was seen by an emergency room physician, and an X-ray revealed no fracture or dislocation. The patient was placed in immobilization and followed up with a sports medicine physician in our office. A magnetic resonance imaging (MRI) of the left knee was ordered and a referral to orthopedics was made. The patient had no history of injury or surgery to the left knee. In the remote past, she underwent ACL reconstruction of the contralateral knee for a soccer injury. She also had a history of deep vein thrombosis after bunion surgery.

On examination, the patient was alert and oriented, answering appropriately. She was non-weight bearing on the injured left side. She had a grade III effusion. Her skin was slightly ecchymotic laterally but otherwise without lesion. On motion examination, she could achieve full extension symmetric to the contralateral knee, however, she had mechanical block to flexion at 90 degrees. She had exquisite pain at the extremes of range of motion, both flexion and extension. The finding of full extension symmetric to the opposite knee was surprising as most BHMT cause some lack of extension. This finding may be caused by lack of return of full extension (hyperextension) of her contralateral knee from her previous ACL reconstruction.

Her anterior drawer test as well as her Lachman examination was $3+$. The posterior drawer was negative and she did not have posterior sag. She had slight laxity with valgus stress and had no varus laxity. The Dial test was negative. The patient had bilateral joint line tenderness and a painful McMurray maneuver. Her calf was soft and supple. She had a normal neurovascular examination distal to the injury.

$\mathrm{X}$-rays were reviewed, and no fracture, dislocation, or other boney pathology was appreciated. The MRI was reviewed and showed the she had suffered an ACL rupture along with buckle-handle tears of both the medial and lateral meniscus. Both of these menisci were displaced and flipped anteriorly and centrally (anterior and lateral for medial BHMT, anterior and medial for lateral BHMT) past their femoral condyles and were lodged in the notch (-Fig. 1). The patient had also suffered a mild injury to the medial collateral ligament.

The patient was scheduled for the operating room and received surgical reconstruction within 1 week of the injury. A diagnostic knee arthroscopy was performed. She ruptured her ACL from the femoral attachment and had bimeniscal buckle-handle tears that were displaced into the femoral notch (-Fig. 2). Both menisci were torn at the "red-red" zone. Therefore, repair of both menisci was performed using an inside-out technique (-Fig. $\mathbf{3}$ ).

For the medial meniscus repair, a posteromedial incision was made just posterior to the medial collateral ligament with one-third of the incision above the joint and two-third of the incision below the joint line. The interval between the posteromedial capsule and the medial head of the gastrocnemius was created and the lower half of a speculum was placed in the interval to protect the neurovascular structures. Using zone-specific single and double lumen cannulas (Acufex Meniscal Stitcher, Smith \& Nephew, Andover, MA), 2-0 Fiberwire meniscal sutures (Arthrex, Naples, FL) were placed from the lateral portal on both the superior and inferior surfaces of the meniscus in a vertical mattress fashion. The sutures were then tied to the capsule with the knee bent of 30 degrees from posterior to anterior.
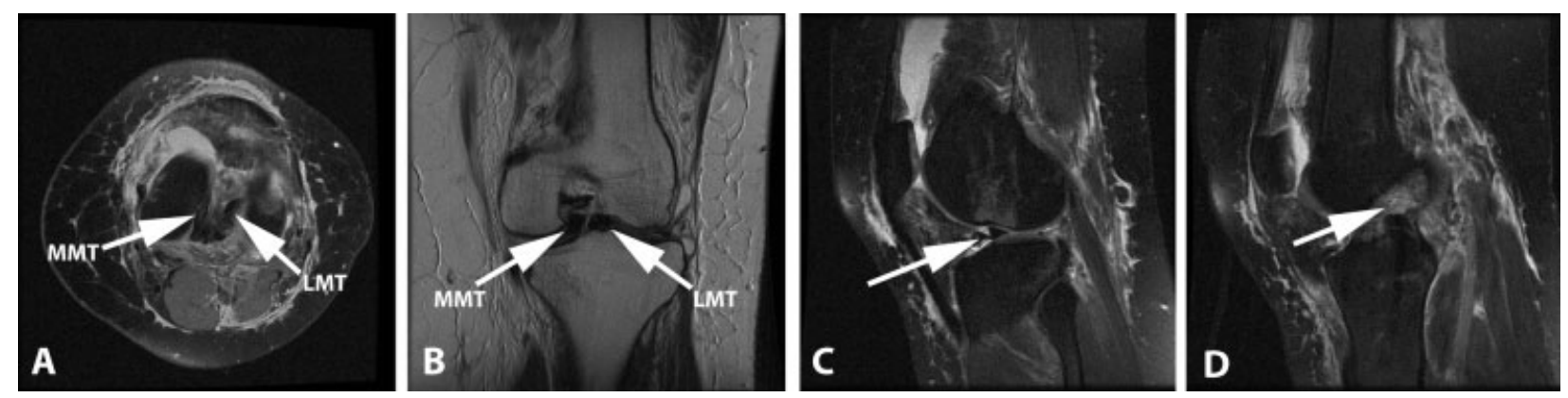

Fig. 1 Axial T2-weighted MRI (A) showing bimeniscal displaced bucket-handle tears in the intercondylar notch (arrows). Coronal T1-weighted MRI (B) showing bimeniscal displaced bucket-handle tears (arrows) with displacement into the intercondylar notch. Sagittal T2-weighted MRI (C) showing a double anterior horn meniscal sign (arrow) indicating a displaced posterior horn meniscal tear. Sagittal T2-weighted MRI (D) showing rupture of the ACL (arrow). $\mathrm{ACL}$, anterior cruciate ligament; LMT, lateral meniscal tear; MMT, medial meniscal tear; MRI, magnetic resonance imaging. 


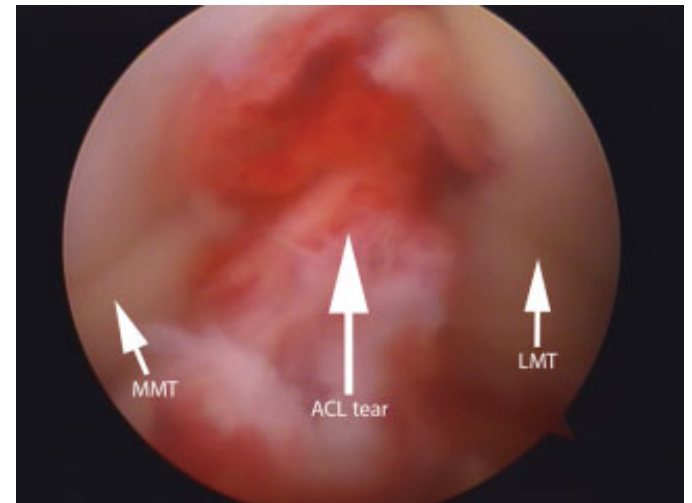

Fig. 2 Arthroscopic view of displaced bimeniscal bucket-handle tears (arrows) with ACL rupture. ACL, anterior cruciate ligament; LMT, lateral meniscal tear; MMT, medial meniscal tear.

Likewise, a posterolateral incision was made just posterior to the lateral collateral ligament. The interval between the iliotibial band and the biceps tendon was developed, followed by placing the bottom half of a speculum between the posterolateral capsule and the lateral head of the gastrocnemius to protect the neurovascular structures. From the medial portal, vertical mattress sutures were placed and tied in a similar technique as the medial meniscus repair.

Following this, an ACL reconstruction was done using a quadrupled hamstring autograft. Endobutton fixation (Smith \& Nephew, Andover, MA) was used on the femur with a screw and sheath used for tibial fixation. The patient tolerated the procedure well and was sent home the same day. Low-molecular-weight heparin was used postoperatively because of her history of previous DVT. A standard rehabilitation protocol from our institution was used, limiting flexion past 90 degrees during the first 4 weeks and protective weight bearing for the first 4 weeks.

Postoperative course for the patient was without complication. She regained full motion by 8 weeks postprocedure. She was able to get back to professional billiards tournaments by 3 months postprocedure. At 2-year follow-up, there was no loss of motion, no joint line tenderness, negative Lachman test and pivot shift, and negative McMurray test. She was back to all the activities with no symptoms.

\section{Discussion}

The yearly incidence of acute meniscal injury has been reported to be as high as 61 per $100,000 .^{13}$ These same authors estimate that 850,000 meniscal procedures are performed annually in the United States. Fewer than $10 \%$ of these injuries are BHMT. ${ }^{4,5}$ We present a rare case of medial and lateral displaced BHMT in a 31-year-old female nonathlete with an acute ACL rupture. To our knowledge, this injury has been described only once in a younger athlete. ${ }^{8}$

MRI is the dominant noninvasive technique for evaluating meniscal lesions of the knee. However, with the displacement of a BHMT it may be difficult to sort out what pathology is present in the knee. Six signs seen on MRI can be helpful in identifying a BHMT. ${ }^{14}$ The absence of the bow tie sign describes the appearance of only one or no meniscal body segment in peripheral sagittal MR slices. A double PCL sign is a displaced meniscal fragment anterior to the PCL on the sagittal MRI giving the appearance of two PCLs. The double anterior horn sign represents when an intact anterior horn and the displaced meniscal fragment lay next to each in the same horizontal planes opposed to a flipped meniscus sign when the anterior horn looks abnormally large. A posterocentrally displaced meniscal fragment can show a disproportional posterior horn sign. A large posterior meniscal horn is seen in the central section than that in the peripheral section on sagittal MRI. Finally, the fragment within the intercondylar notch sign denotes a flipped meniscal fragment in the notch. The two most common signs seen are the fragment in the notch and the absent bow tie sign. ${ }^{8}$

Once we determine that a BHMT exists, can we accurately predict that the tear can be repaired? Thoreux et al ${ }^{7}$ retrospectively reviewed 28 patients who underwent knee arthroscopy and were diagnosed with a BHMT at the time of surgery. Two radiologists, who were unaware of the intraoperative findings or patient history, reviewed the MRI and predicted reparability of the BHMT when all the three of the following criteria were met: (1) A bucket-handle rim segment less than $4 \mathrm{~mm}$ wide; (2) tear length of $1 \mathrm{~cm}$ or greater, regardless of the total lesion length, and; (3) minimal damage to the inner and peripheral meniscal fragments. These predictions were then compared with what procedure was performed at the time of arthroscopy. The MRI prediction of reparability agreed with arthroscopy in 26 of 28 BHMTs
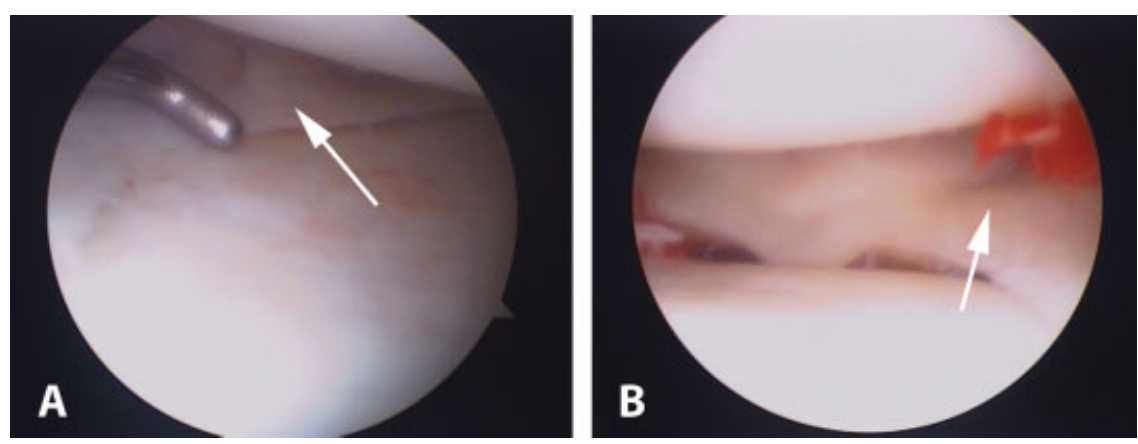

Fig. 3 Arthroscopic views of the repaired (A) medial meniscus and (B) lateral meniscus (arrows). 
(92.9\%). The patient in this study met all the three MRI criteria for reparability, which correlated with the arthroscopic findings.

In our case, the patient had an associated ACL rupture. The frequency of association of BHMTs and ACL rupture varies in the literature. In a study of 36 BHMTs by Ververidis et al, ${ }^{14}$ an associated ACL rupture was found in $44.4 \%$ of the cases. This is much higher than the $11 \%$ reported by Shakespeare and Rigby. ${ }^{15}$ In 39 patients with BHMTs, Wright et $\mathrm{al}^{4}$ found an associated ACL rupture in $48 \%$ of the cases. No bimeniscal bucket-handle tears were reported by any of these authors. This makes our case report unique. Because of this concomitant injury, the success rate of healing of the meniscal repair is higher compared with isolated tears. Canon reported a satisfactory result of $83 \%$ in patients treated along with ACL reconstruction. ${ }^{16}$ This is in contrast to $75 \%$ satisfaction in isolated meniscal tears. These results were also supported by Gill and Diduch, who reported on 32 patients with meniscal repair and ACL reconstruction. ${ }^{17}$ At 2-year follow-up, 91\% had a good outcome.

This is a case of a 31-year-old female patient with bimeniscal bucket-handle tears with an associated ACL rupture who received ligament reconstruction and repair of her menisci, demonstrating the typical signs and symptoms of a displaced BHMT as well as imaging and arthroscopic pictures. Family physicians and orthopedists alike may be unfamiliar with this diagnosis, which is extremely rare in the younger patients. As this case demonstrates, MRI is essential for the proper diagnosis of a BHMT, as it is the most reliable predictor of surgical repairability.

\section{Disclosures}

David C. Flanigan is on the speaker's bureau for Sanofi and Smith \& Nephew. The authors have no conflicts of interest to disclose.

\section{References}

1 Cipolla M, Scala A, Gianni E, Puddu G. Different patterns of meniscal tears in acute anterior cruciate ligament (ACL) ruptures and in chronic ACL-deficient knees. Classification, staging and timing of treatment. Knee Surg Sports Traumatol Arthrosc 1995; 3(3):130-134
2 Finsterbush A, Frankl U, Matan Y, Mann G. Secondary damage to the knee after isolated injury of the anterior cruciate ligament. Am J Sports Med 1990;18(5):475-479

3 Keene GC, Bickerstaff D, Rae PJ, Paterson RS. The natural history of meniscal tears in anterior cruciate ligament insufficiency. Am J Sports Med 1993;21(5):672-679

4 Wright DH, De Smet AA, Norris M. Bucket-handle tears of the medial and lateral menisci of the knee: value of MR imaging in detecting displaced fragments. AJR Am J Roentgenol 1995;165(3): 621-625

5 Magee TH, Hinson GW. MRI of meniscal bucket-handle tears. Skeletal Radiol 1998;27(9):495-499

6 Greis PE, Bardana DD, Holmstrom MC, Burks RT. Meniscal injury: I. Basic science and evaluation. J Am Acad Orthop Surg 2002;10(3): 168-176

7 Thoreux P, Réty F, Nourissat G, et al. Bucket-handle meniscal lesions: magnetic resonance imaging criteria for reparability. Arthroscopy 2006;22(9):954-961

8 Tecklenburg K, Schoepf D, Hoser C, Fink C. Anterior cruciate ligament injury with simultaneous locked bucket-handle tears of both medial and lateral meniscus in a 19-year-old female professional ski racer: a case report. Knee Surg Sports Traumatol Arthrosc 2007;15(9):1125-1129

9 Bosch U. Combined injury of the medial and lateral meniscus and the anterior cruciate ligament [in English, German]. Oper Orthop Traumatol 2006;18(5-6):485-489

10 Brammer H, Sover E, Erickson S, Stone J. Simultaneous identification of medial and lateral bucket-handle tears: the Jack and Jill lesion. AJR Am J Roentgenol 1999;173(3):860-861

11 Cetik O, Cirpar M, Eksioglu F, Uslu M. Simultaneous bucket handle tear of both medial and lateral menisci of a knee with chronic anterior cruciate ligament deficiency. Knee Surg Sports Traumatol Arthrosc 2006;14(4):356-359

12 Bugnone AN, Ramnath RR, Davis SB, Sedaros R. The quadruple cruciate sign of simultaneous bicompartmental medial and lateral bucket-handle meniscal tears. Skeletal Radiol 2005;34(11):740-744

13 Baker BE, Peckham AC, Pupparo F, Sanborn JC. Review of meniscal injury and associated sports. Am J Sports Med 1985;13(1):1-4

14 Ververidis AN, Verettas DA, Kazakos KJ, Tilkeridis CE, Chatzipapas $\mathrm{CN}$. Meniscal bucket handle tears: a retrospective study of arthroscopy and the relation to MRI. Knee Surg Sports Traumatol Arthrosc 2006;14(4):343-349

15 Shakespeare DT, Rigby HS. The bucket-handle tear of the meniscus. A clinical and arthrographic study. J Bone Joint Surg Br 1983;65(4): 383-387

16 Canon WDJr. Arthroscopic meniscal repair. In: McGinty JB, Caspari RB, Jackson RW, Poehling GG, eds. Operative Arthroscopy. 2nd ed. Philadelphia, PA: Lippincott-Raven; 1996:299-315

17 Gill SS, Diduch DR. Outcomes after meniscal repair using the meniscus arrow in knees undergoing concurrent anterior cruciate ligament reconstruction. Arthroscopy 2002;18(6):569-577 